\title{
Evaluation of Patients' Satisfaction with Health Services Provided in Golestan Hospital, Tehran
}

\section{ART ICLE INF O}

\section{Article Type}

Descriptive Study

\section{Authors}

Pirooz F* $M P H, M D$,

Nuri M. ${ }^{1} P h D$,

Goli M. ${ }^{2} M S c$

\section{How to cite this article}

Pirooz F, Nuri M, Goli M. Eval-

uation of Patients' Satisfaction with Health Services Provided in Golestan Hospital, Tehran. Iranian Journal of War \& Public Health. 2018;10(3):143-149.
*"Aging Research Center", "Aging Department, Social Science Faculty", University of Social Welfare \& Rehabilitation Sciences, Tehran, Iran ${ }^{1}$ Infectious Diseases Department, Medicine Faculty, Army Medical University, Tehran, Iran

${ }^{2}$ Anthropology Department, Medicine Faculty, Army University of Medical Sciences, Tehran, Iran

\section{Correspondence}

Address: Aging Department, Social Science Faculty, University of Social Welfare \& Rehabilitation Sciences, Koudakyar Street, Evin, Tehran, Iran. Postal Code: 1668619551

Phone: $+98(21) 22180004$

Fax: +98 (21) 22542796

fereshtehpirooz@gmail.com

\section{Article History}

Received: August 08, 2017

Accepted: February 24, 2018

ePublished: August 29, 2018

\section{A B S T R A C T}

Aims Patient satisfaction with the services provided during hospitalization is one of the important indices of the quality of health care centers. The aim of this study was to evaluate patients' satisfaction in Golestan Hospital in Tehran.

Instruments \& Methods In this cross sectional, descriptive-analytical study, 426 patients (8 to 96 years old) were selected from patients hospitalized in Golestan Hospital (Tehran) with duration of stay of more than one day, using available sampling method. The study period was one year from April 2016 to March 2017. The questionnaire consisted of two parts: demographic and proprietary information. The standard checklist for patients' satisfaction from the health system development plan was used to set specific questions. The data were analyzed, by SPSS 16 software, using ANOVA, Chi-square, and independent t-tests.

Findings $73.82 \%$ (314 people) of the patients were satisfied with the services provided at the center and $12.65 \%$ (54 people) were dissatisfied. The highest satisfaction was in medical services (91.37\%) and the lowest was related to food situation (60.55\%). The variable of gender did not have significant relationship with satisfaction component $(\mathrm{p}=0.052)$, but marital status $(p=0.004)$, level of education $(p<0.001)$ and age $(p=0.002)$ had significant relationship with satisfaction component.

Conclusion Marital status, level of educational and age are related to the degree of satisfaction of hospitalized patients, but gender is not related to satisfaction.

Keywords Satisfaction; Health Services; Inpatient

\section{CIT A T I O N L IN KS}

[1] Patient satisfaction in emergency medicine [2] Satisfaction with care [3] Satisfaction rate of hospitalized patients in military hospitals from presented services [4] Quality management: Philosophy and trend [5] A discipline-specific determination of high quality nursing care [6] Study of the satisfaction rate of hospitalized patients at Ilam hospitals [7] Patient satisfaction from pre and postoperative nursing care in hospitals affiliated to Babol University of Medical Sciences [8] Patient perceptions of physician empathy, satisfaction with physician, interpersonal trust, and compliance [9] Iranian Health Perception Survey (IHPS): The study protocol [10] Patients' satisfaction of medical services in Qazvin education hospitals [11] Validation of the EORTC QLQ-SAT32 cancer in patient satisfaction questionnaire by selfversus interview-assessment comparison [12] Patient satisfaction with nursing care in the context of health care: A literature study [13] Development of a questionnaire to measure patients' satisfaction with general practitioners' services [14] Assessment of Patient Satisfaction from Nursing Care in Hospitals of Iran University of Medical Sciences [15] Assessment of patient satisfaction with nursing services provided in general wards of university hospitals in Bushehr 2012 [16] Health system transformation project, an opportunity or a threat for doctors (Editorial) [17] A comparative study on patient satisfaction with hospital services in Amol [18] Relationship between nurse caring behaviors from patients' perspectives \& their satisfaction [19] Patients' level of satisfaction on quality of health care at Mwananyamala hospital in Dar es Salaam [20] Inpatient satisfaction with health system transformation project in Mazandaran educational hospitals' Iran [21] Relationship between moral distress and job satisfaction among nurses of Tehran University of Medical Sciences hospitals [22] Patient Satisfaction with Nursing Care in Behshahr Public Hospitals, Iran [23] Who is responsible for health: Iranian Health Perception Survey (IHPS) [24] Patients satisfaction of medical care delivered in outpatient clinics of endocrinology and metabolism in Tehran, Iran [25] Patients' Satisfaction with Services Provided at the Center for Breast Disease 


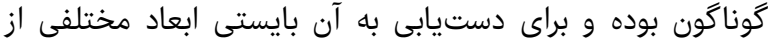

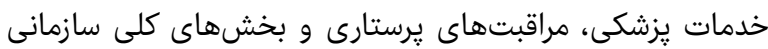

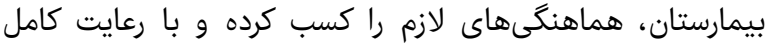

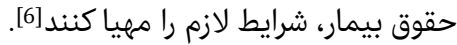

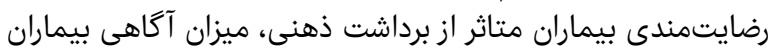

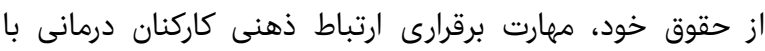

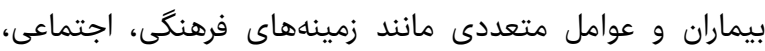

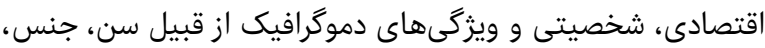

و سطح تحصيلات است [7].

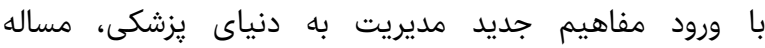

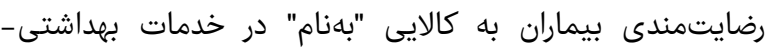

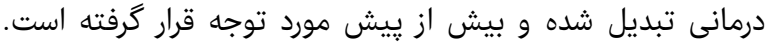

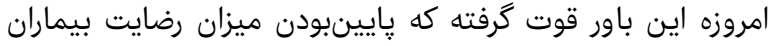

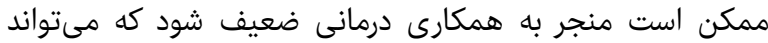

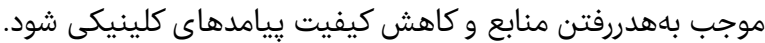

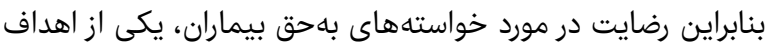

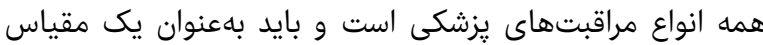

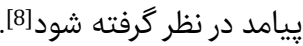

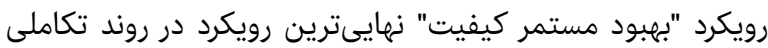

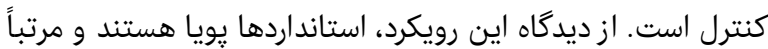

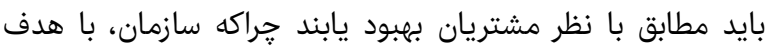

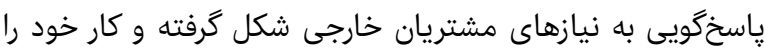

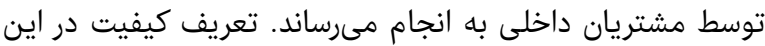

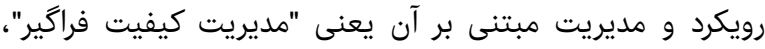

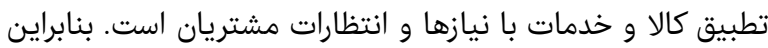

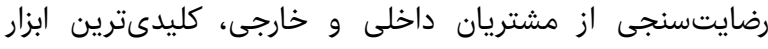

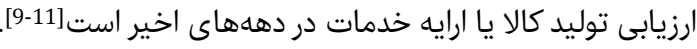

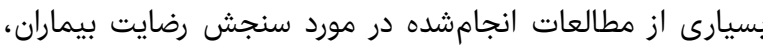

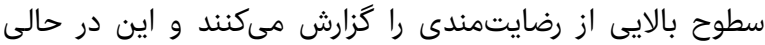

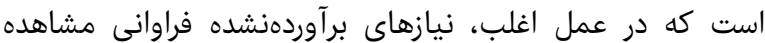

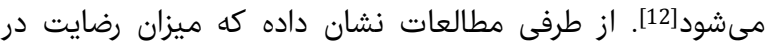

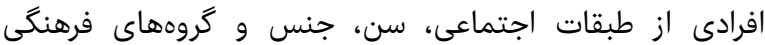

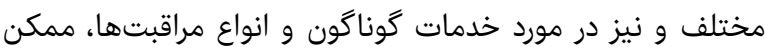

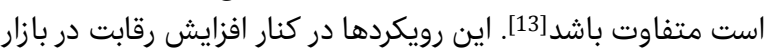

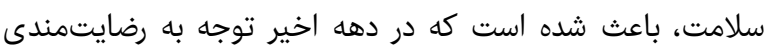

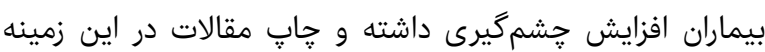

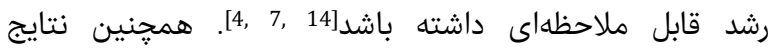
نظرسنجىها نشان داده است، علىرغم اينكه يرستاران مسئول إرائ ارايه

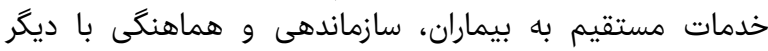

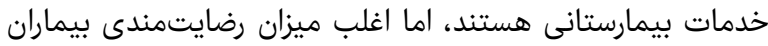

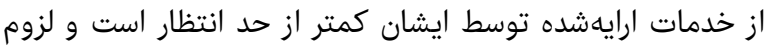

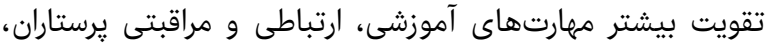

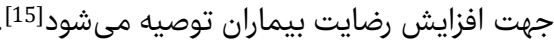

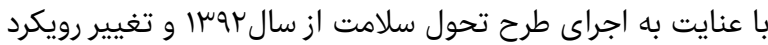

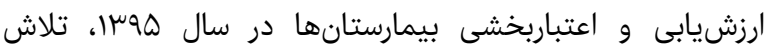

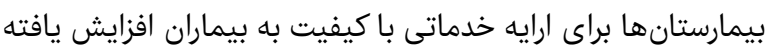

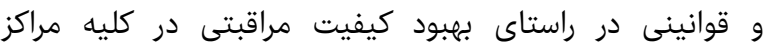

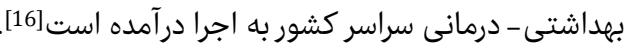

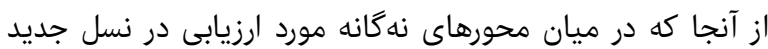

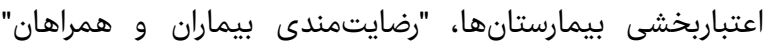

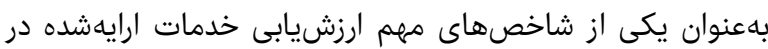

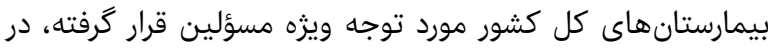

\section{رضايتسنجى بيماران بسترى از خدمات بهداشتى - درمانى ارايهشده در بيمارن بستريتان

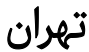

\section{MPH, MD "فرشته يِيروز}

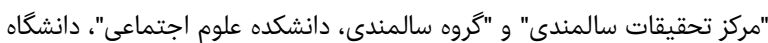
علوم بهزيستى و توانبخشى، تهران، ايران

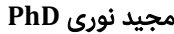

كروه بيمارى هاى عفونى، دانشكده يزشكى، دانشكاه علوم يزشكى ارتش، تهران،

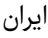

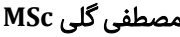

كروه طب رزم، دانشكده يزشكى، دانشكاه علوم يزشكى ارتش، تهران، ايران

جكيده

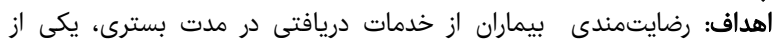

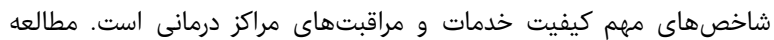
حاضر با هدف رضايتسنجى بيماران بسترى در بيمارستان گلستان تران تهران انجام

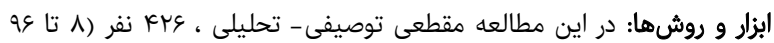

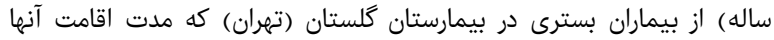

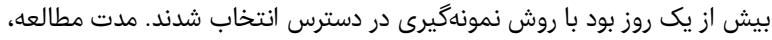

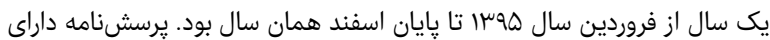

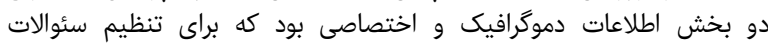

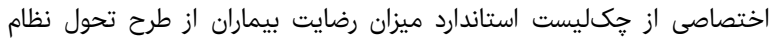

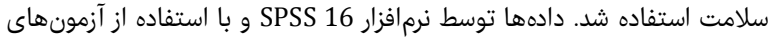

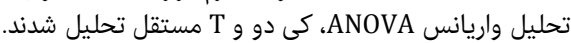

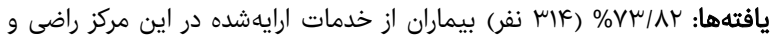

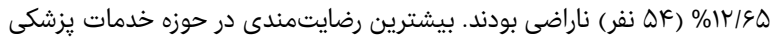

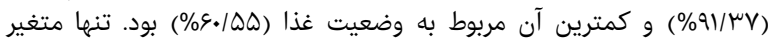

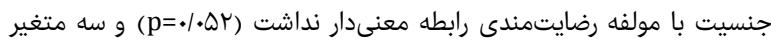

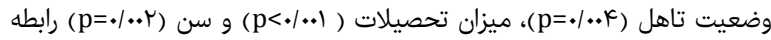

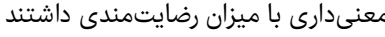

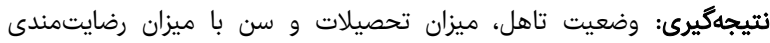

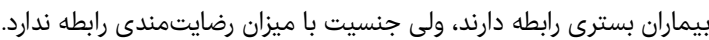
كليدواثرها: رضايتمندى، خدمات بهداشتى درمانى، بيماران بسترى

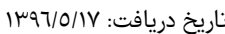

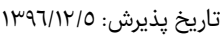

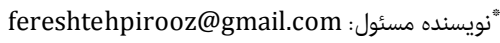

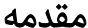

رضايت بيماران معيار مناسبى براى ارزيابى كيفيت خدمات درواني ترانى

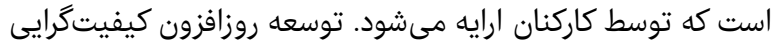

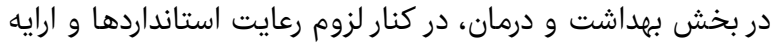

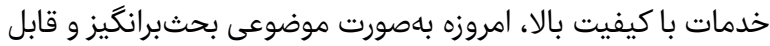

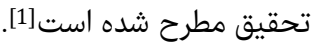

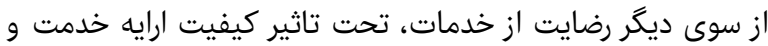

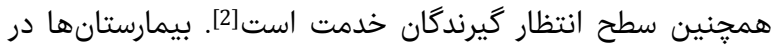

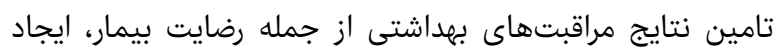

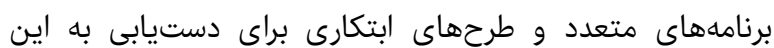

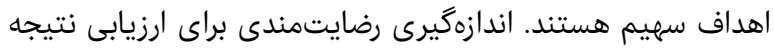

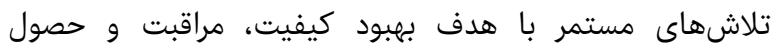

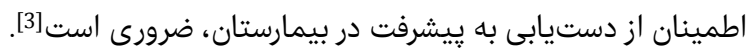

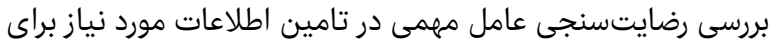

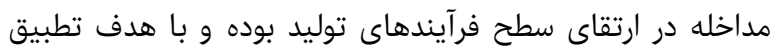

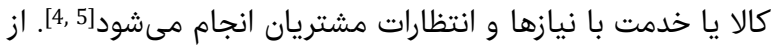

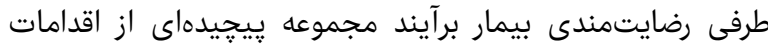




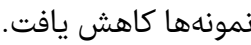

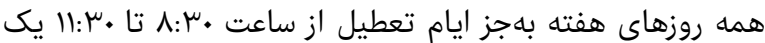

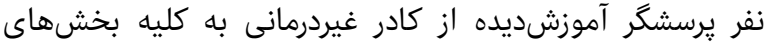

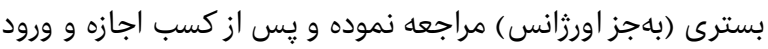

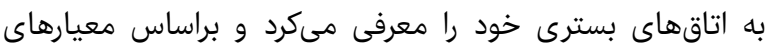

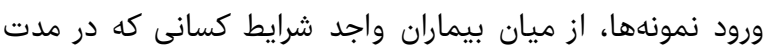

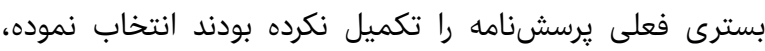

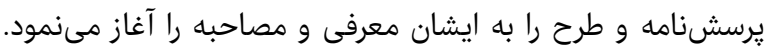

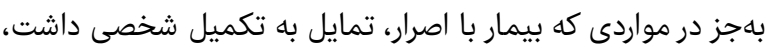

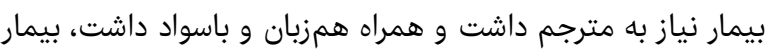

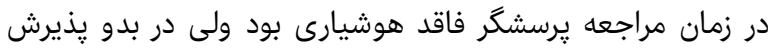

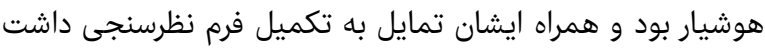

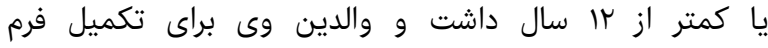

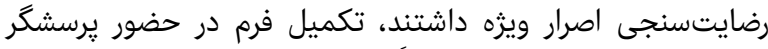

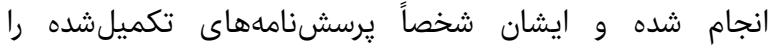
جمعآورى مى نمود.

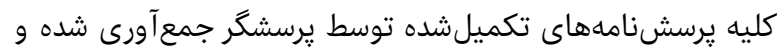

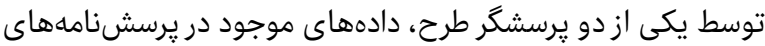

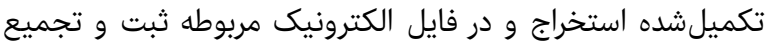

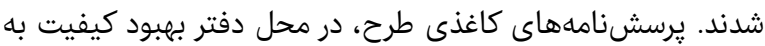

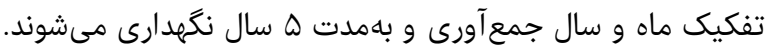

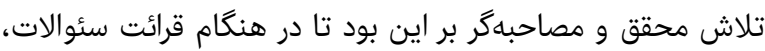

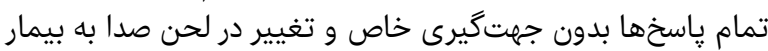

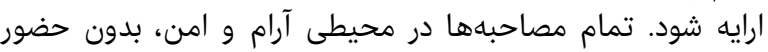

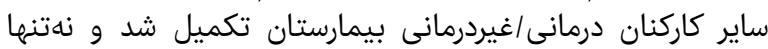

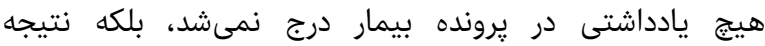

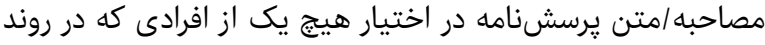

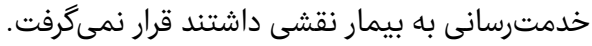

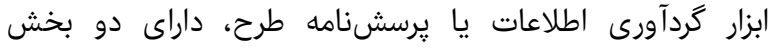

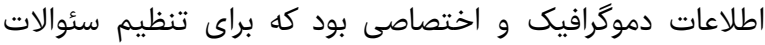

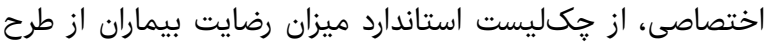

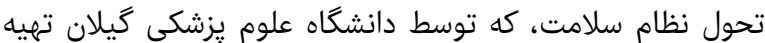

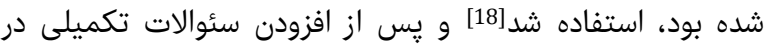

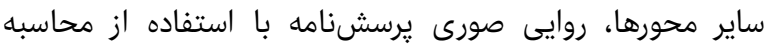

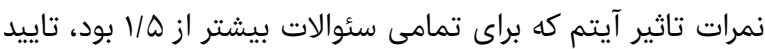

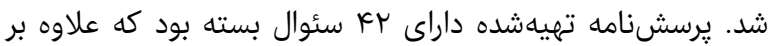

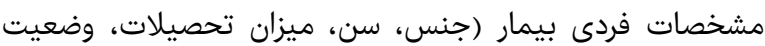

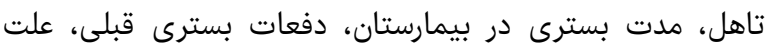

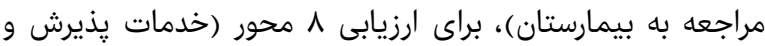

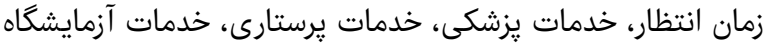

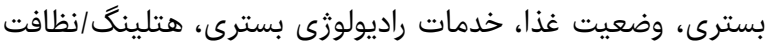

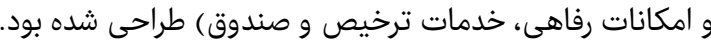

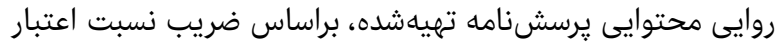

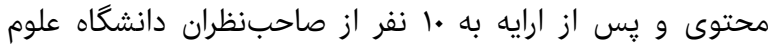

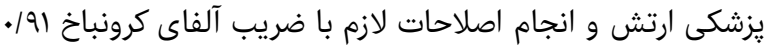

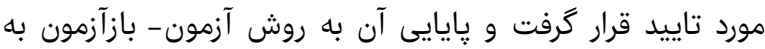

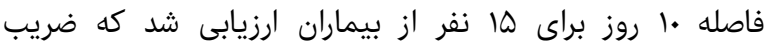

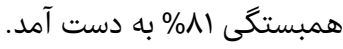

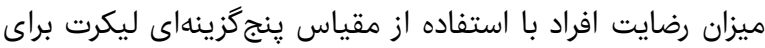

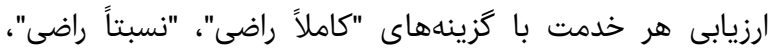

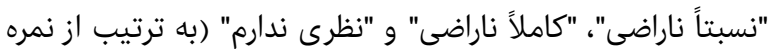

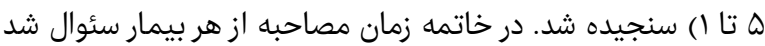

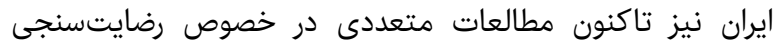

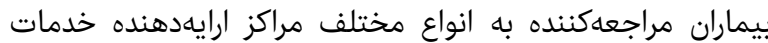

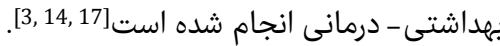

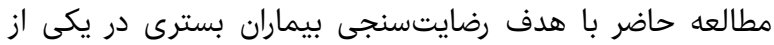

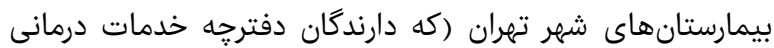

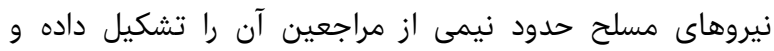

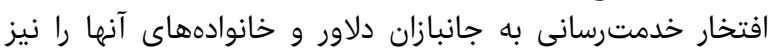

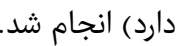

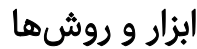

اين مطالعه از نوع مقطعى و توصيفى - تحليلى بوده و و جمعيت

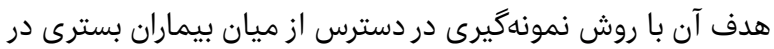

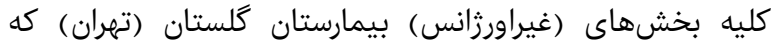

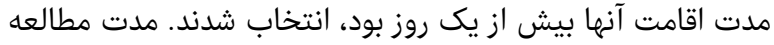

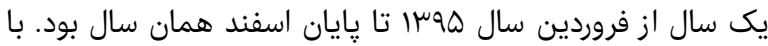

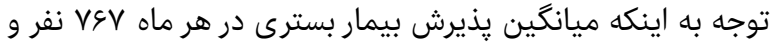

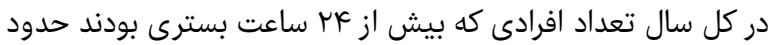

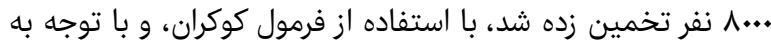

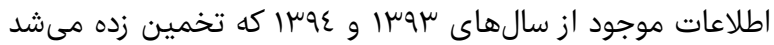

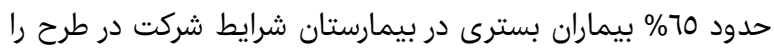

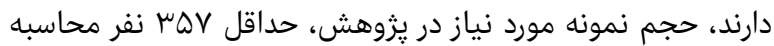

در نهايت، شب K بيمار در مطالعه شركت نموده و به سئوالات

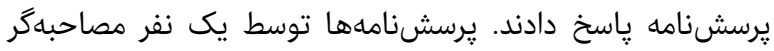

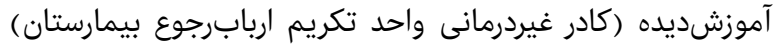

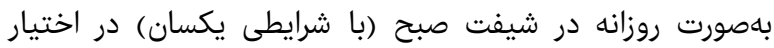

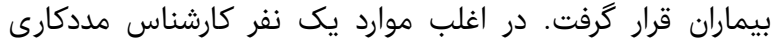

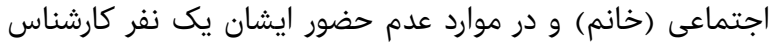

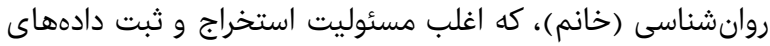

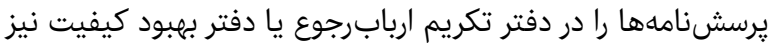

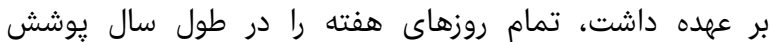

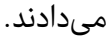
معيارهاى ورود به مطالعه ماده عبارت از بسترى حداقل بهمدت

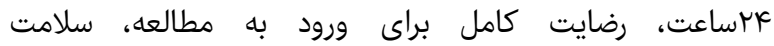

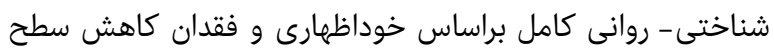

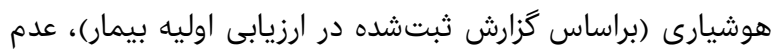

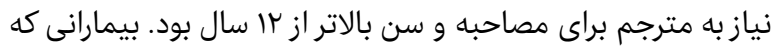

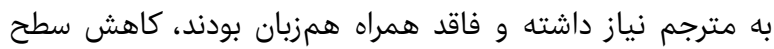

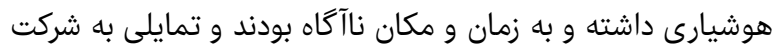

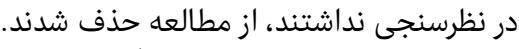

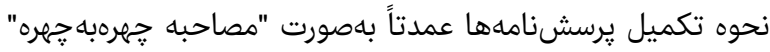

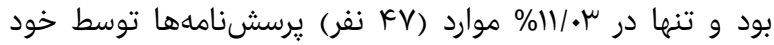

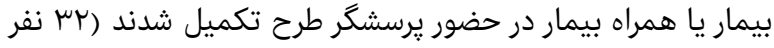

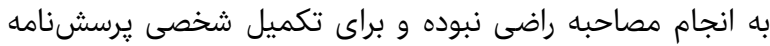

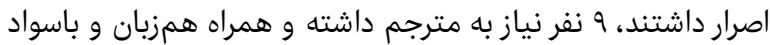

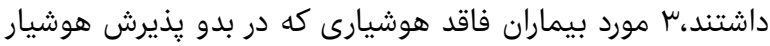

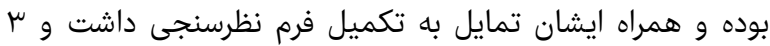

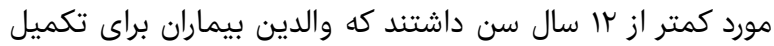

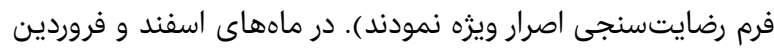

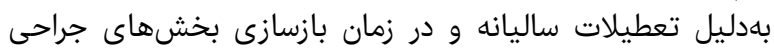

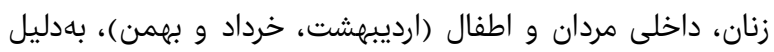

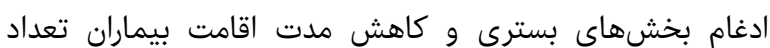


از هتلينگ (امكانات رفاهى، وضعيت نظافت اتاق/تخت/ البسه و

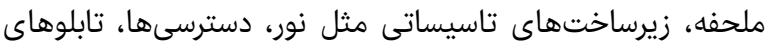

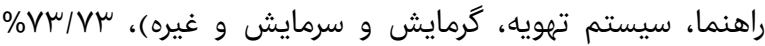

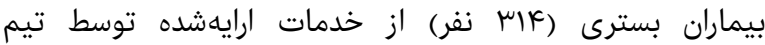

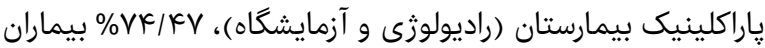

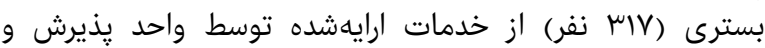

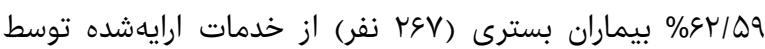
واحد ترخيص و صندوق بيمارستان رضايت داشتند (نمره

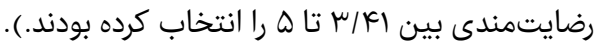

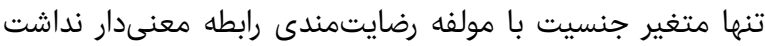

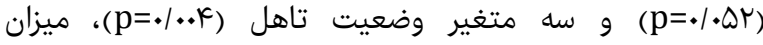

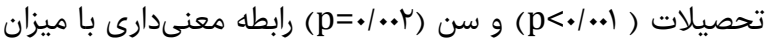

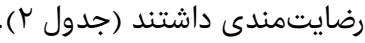

جدول r) توزيع فراوانى متغيرهاى دموگرافيك و رابطه آنها با متوسط دران

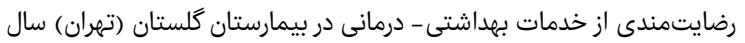
$1 \% 90$

\begin{tabular}{|c|c|c|c|}
\hline معنىدارى & رضوسط درصدى & تعداد (درصد) & متغيرها \\
\hline \multirow{3}{*}{$\cdot / . r r$} & $\mu F / \Delta \Delta \pm \Psi / \| F$ & $\mu \cdot(V / \cdot)$ & كمتر از rا سال \\
\hline & $৭ 9 / 9 \vee \pm 11 / r \Delta$ & $1 . r(r \mu / Q)$ & rا تا qr سال \\
\hline & $\begin{array}{l}V Y / Y G \pm \varepsilon / q \mu \\
\Lambda \varepsilon / V I \pm \Lambda / F V\end{array}$ & $\begin{array}{l}\mid \mu \mu(\mu / / \mu) \\
|\varnothing|\left({ }^{\mu} V / \Lambda\right)\end{array}$ & •ع سال و بيشتر سال \\
\hline.$/ . \Delta r$ & $\begin{array}{l}V \cdot / \cdot r \pm \mid r / F r \\
V|/ r q \pm q / \mu|\end{array}$ & $\begin{array}{l}r \mu V(\Delta Q / \varepsilon) \\
\wedge q(K F / F)\end{array}$ & زنسيت \\
\hline.$/ . * k$ & 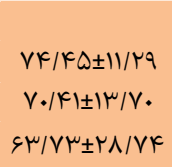 & $\begin{array}{l}I r V(r r / r) \\
r \cdot V)(r \wedge / \varepsilon) \\
\wedge r(I Q / r)\end{array}$ & مجاهرد \\
\hline$<\cdot / . .1$ & 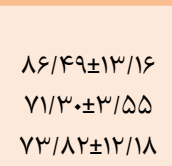 & $\begin{array}{l}|Y|(Y N / K) \\
19 Y(K G / Y) \\
1.1(Y Q / K)\end{array}$ & دير ديلم دييلم فحصيلات \\
\hline
\end{tabular}

بحث

بيمارستان كلستان علاوه بر اينكه كليه خدمات تشخيصى - درمانى

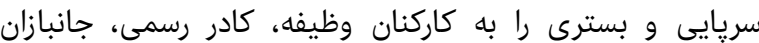

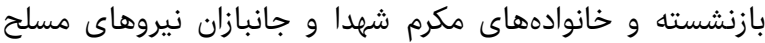

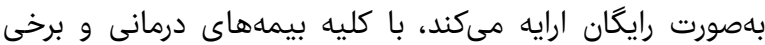

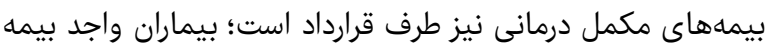

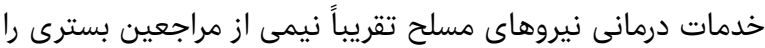

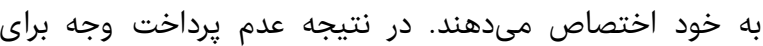

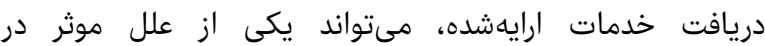

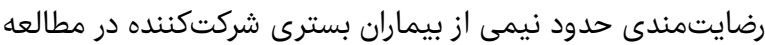

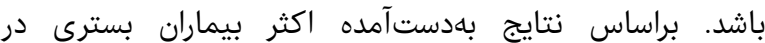

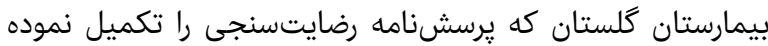

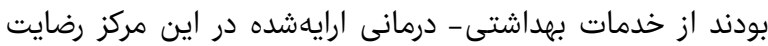

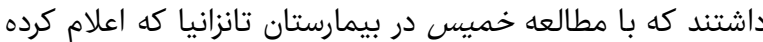

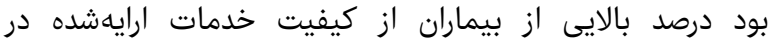

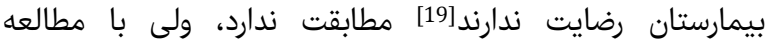

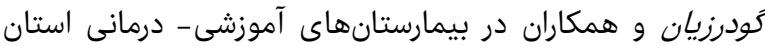

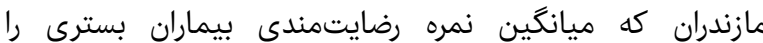

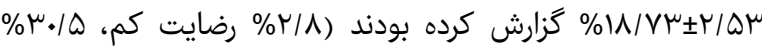

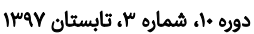

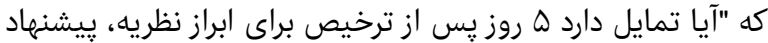

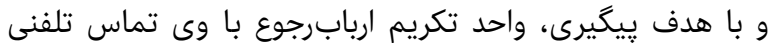

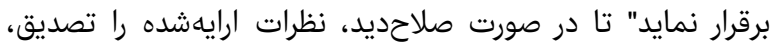

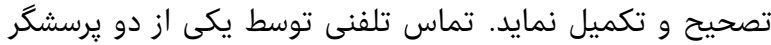

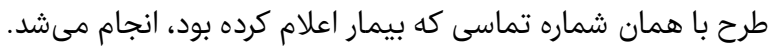

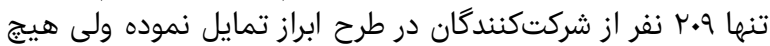

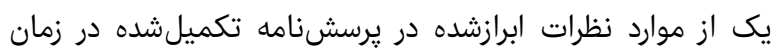
بسترى را تغيير ندادند.

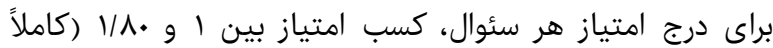

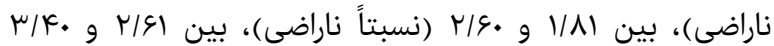

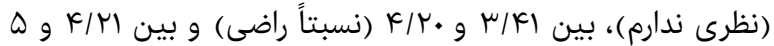

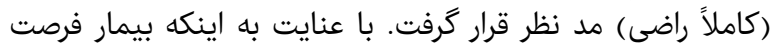

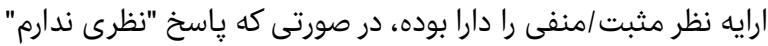

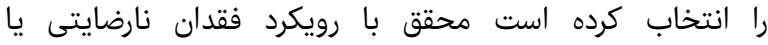

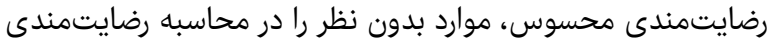

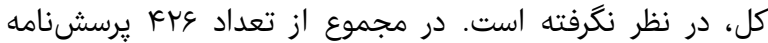

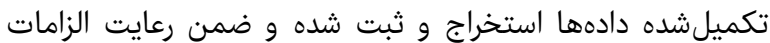

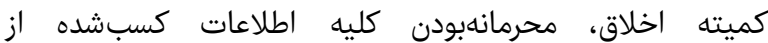
شركتكنندگان در يزوهش تضمينه

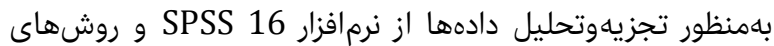

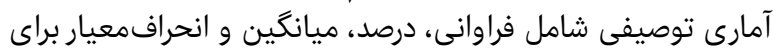

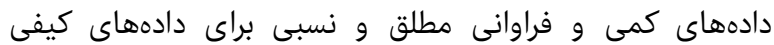

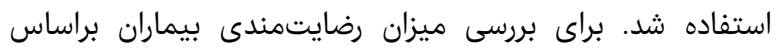

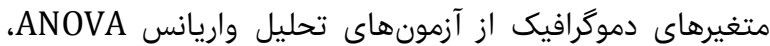
كى دو و T مستقل استفاده شد.

\section{يافتهها}

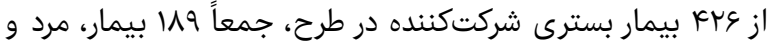

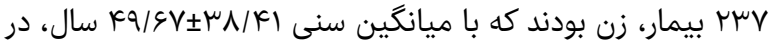

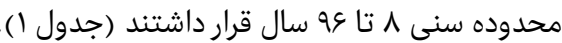

جدول () ميانگين سنى و ميزان رضايتمندى افراد تحت مطالعه در بيمارستان

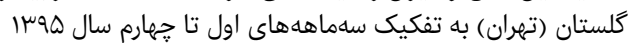

\begin{tabular}{|c|c|c|c|c|c|c|c|}
\hline \multirow{2}{*}{ بذر } & \multirow{2}{*}{ نارضايتى } & \multirow{2}{*}{ رضايتمندر } & \multirow{2}{*}{ سيانكين } & \multirow{2}{*}{ نمونهاد } & \multicolumn{2}{|c|}{ جنس } & \multirow{2}{*}{ سهاماهـ } \\
\hline & & & & & مرد & زن & \\
\hline $\mid K / Q F$ & $1 \% / 9 k$ & $\Delta r / V I$ & $\Delta K / \cdot \cdot \pm K F / \Delta K$ & KV & $r q$ & 11 & اول \\
\hline $18 / 19$ & IF/we & $K Q / 99$ & $\Delta \cdot / \cdot \cdot \pm K I / K V$ & 10 . & $\wedge 1$ & 99 & دوم \\
\hline r & $11 / 90$ & $s F / 9 V$ & $\langle\wedge / \cdot \cdot \pm \mu \cdot / q \Lambda$ & 198 & 90 & vi & سوم سوم \\
\hline • & 1.194 & mF/Aq & $\kappa \& / 98 \pm{ }^{\mu} \Lambda / \Lambda \varphi$ & $\varepsilon \mu$ & rr & $\mu$ & جهارم \\
\hline $\mid \mu / \Delta \mu$ & $\mid r / \& D$ & $\Lambda r / V \mu$ & $k q / \varepsilon V \pm \mu \nu / k 1$ & krs & $r \mu v$ & 119 & جمع كل \\
\hline
\end{tabular}

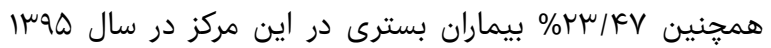

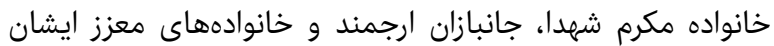

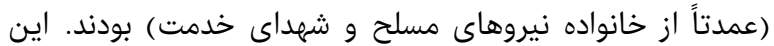

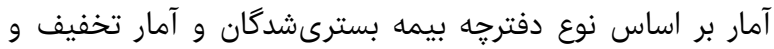

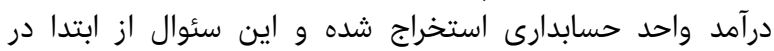

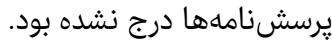

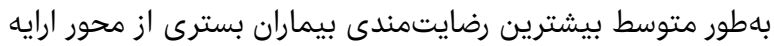

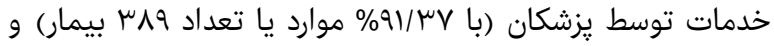

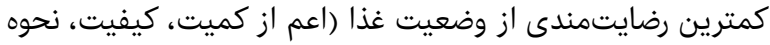

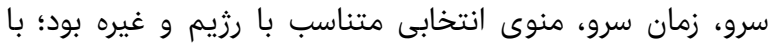

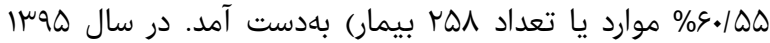

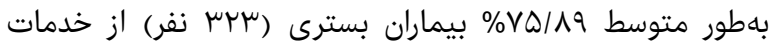

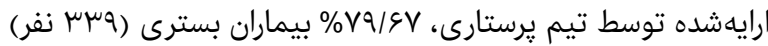

فصلنامه علمى - بُزوهشى طب جانباز 


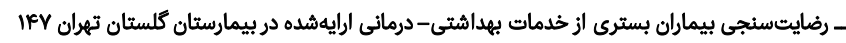

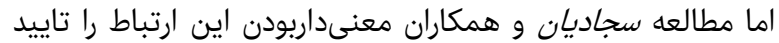
نمود[25].

در مطالعه ما وضعيت تاهل با ميزان رضايتمندى اردار ارتباط

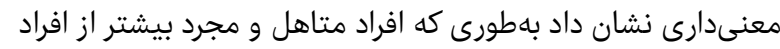

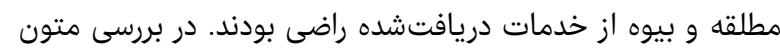

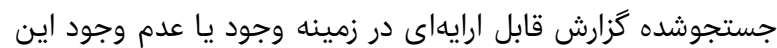

ارتباط يافت نشد.

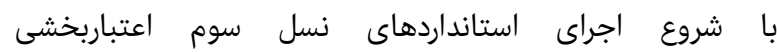

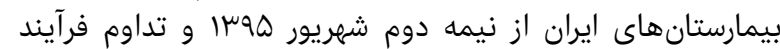

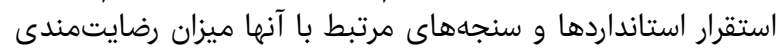

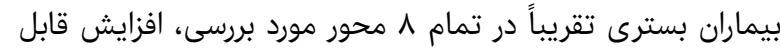

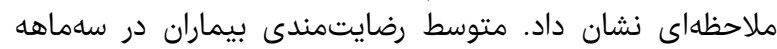

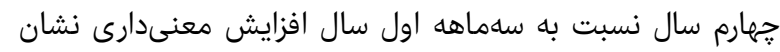

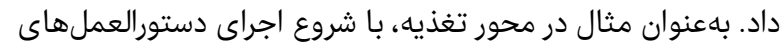

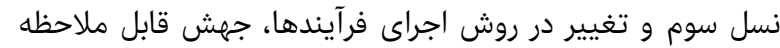

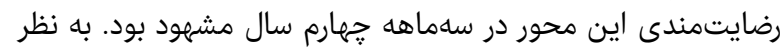

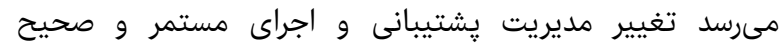

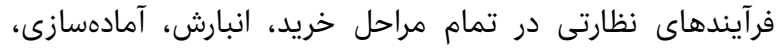

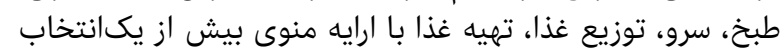

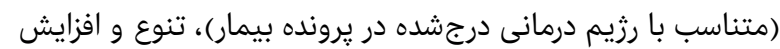

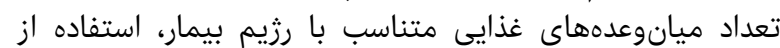

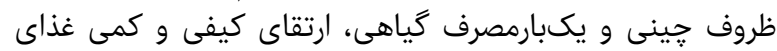

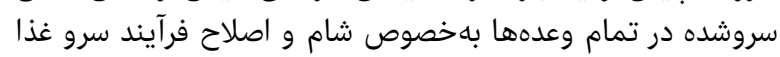

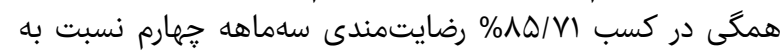
\%هr/VA

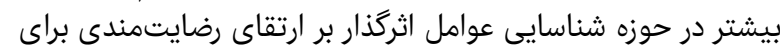

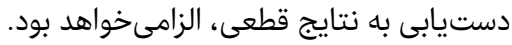

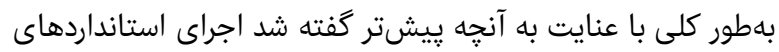

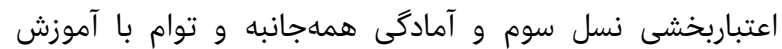

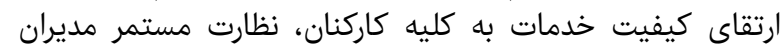

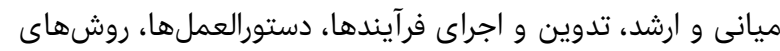

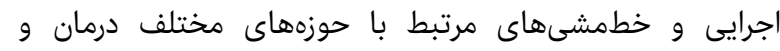

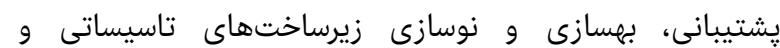

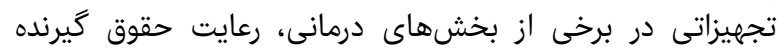

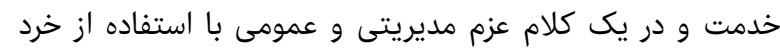

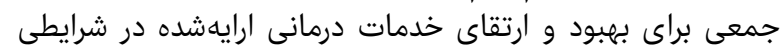

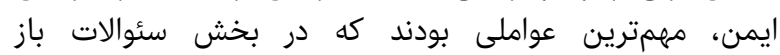

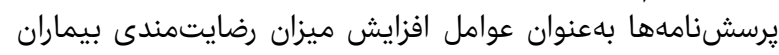

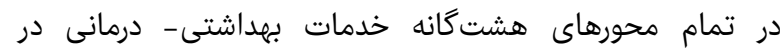

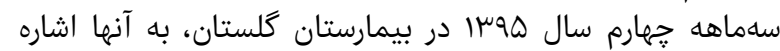

شده بود.

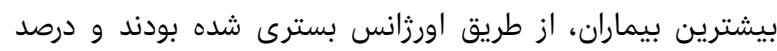

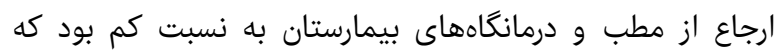

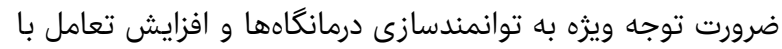

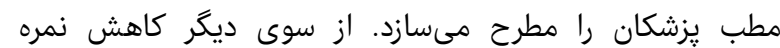

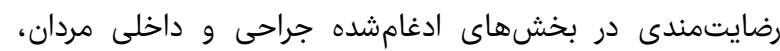

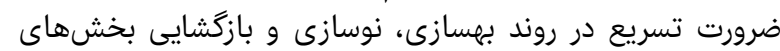

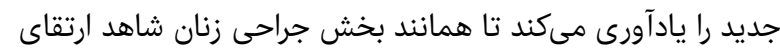

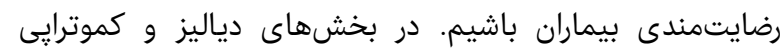

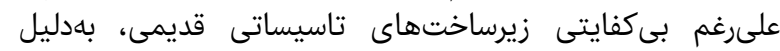

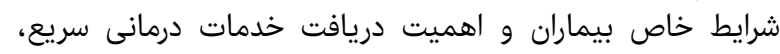

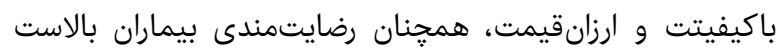

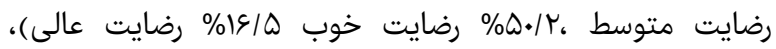

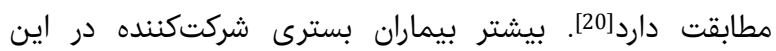

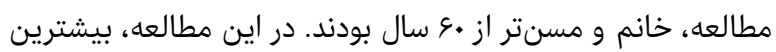

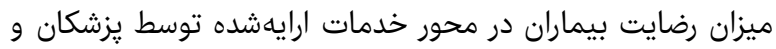

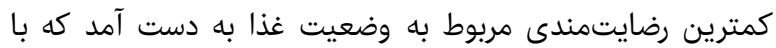

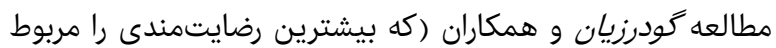

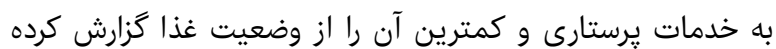

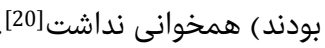

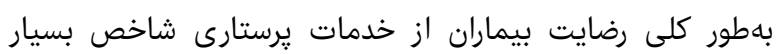

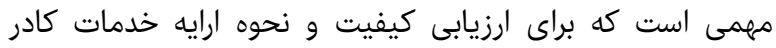

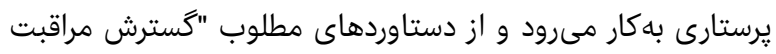

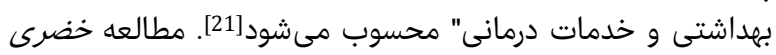

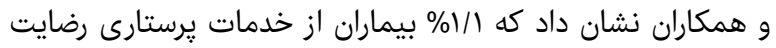

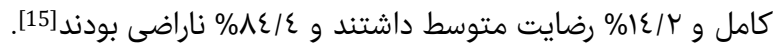

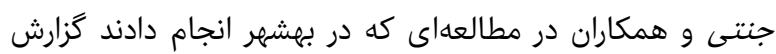

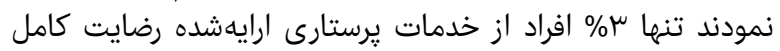

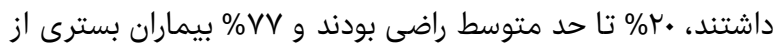

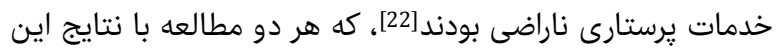
مطالعه انطباق نداشتند.

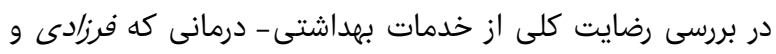

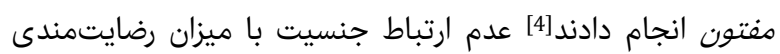

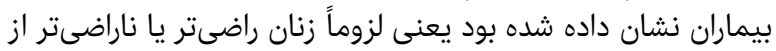

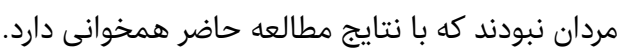

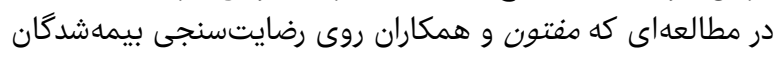

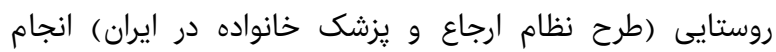

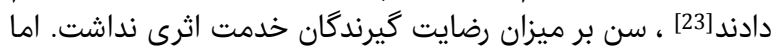

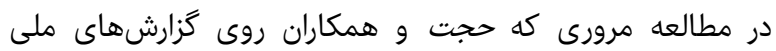
كشورهاى مختلف در خصوص رضايت بيماران بسترى انجام دادي دادند،

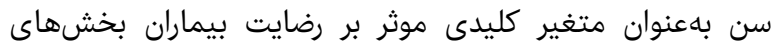

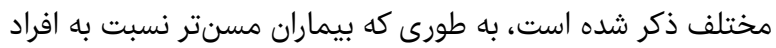

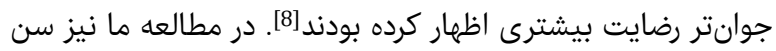

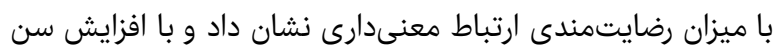

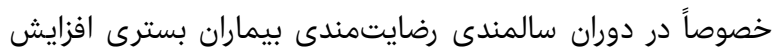

يافت. در مطالعه حاضر، ميزان تحصيلات با رضايتمندى از خدان خدمات

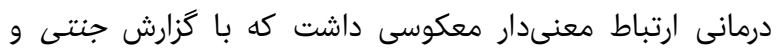

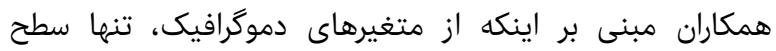

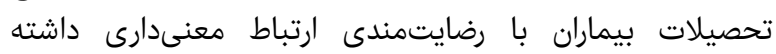

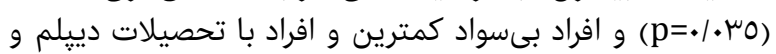

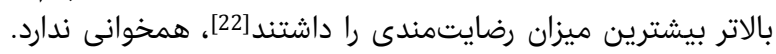

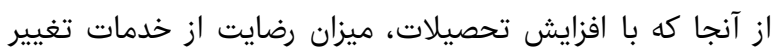

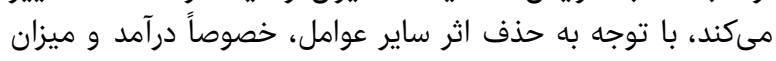

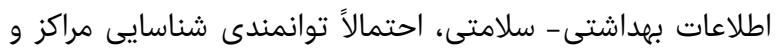

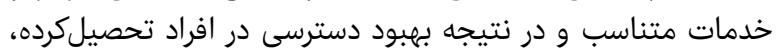

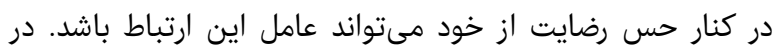

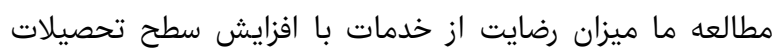

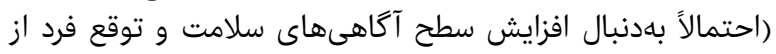

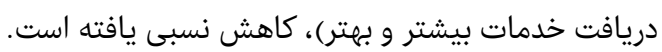

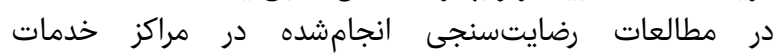

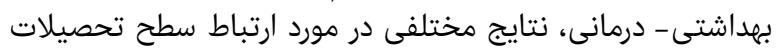

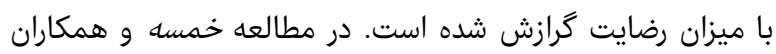

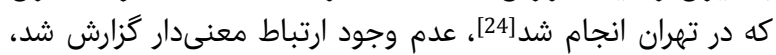


of hospitalized patients at Ilam hospitals. J Ilam Univ Med Sci. 2004;12(3-4):10-16. [Persian]

patients in military hospitals from presented services. J Mil Med. 2010;12(2):101-5.

7- Mortazavi Y, Khalipour Marzoni A, Rabiee O, Rohi Z, Ghomi S. Patient satisfaction from pre and postoperative nursing care in hospitals affiliated to Babol University of Medical Sciences. Preventive Care Nurs Midwifery J. 2013;3(1):38-45. [Persian]

8- Hojat M, Louis DZ, Maxwell K, Markham F, Wender R, Gonnella JS. Patient perceptions of physician empathy, satisfaction with physician, interpersonal trust, and compliance. Int J Med Educ. 2010;1:83-7.

9- Montazeri A, Omidvari S, Azin A, Aeenparast A, Jahangiri K, Sadighi J, et al. Iranian Health Perception Survey (IHPS): The study protocol. Payesh. 2011;10(3):315-22. [Persian]

10- Sheikhi M, Javadi A. Patients' satisfaction of medical services in Qazvin education hospitals. J Qazvin Univ Med Sci. 2004;7(5):62-6. [Persian]

11- Brédart A, Mignot V, Rousseau A, Dolbeault S, Beauloye N, Adam V, et al. Validation of the EORTC QLQSAT32 cancer in patient satisfaction questionnaire by self-versus interview-assessment comparison. Patient Educ Couns. 2004;54(2):207-12

12- Johansson P, Oléni M, Fridlund B. Patient satisfaction with nursing care in the context of health care: A literature study. Scand J Caring Sci. 2002;16(4):337-44.

13- Grogan S, Conner M, Willits D, Norman P. Development of a questionnaire to measure patients satisfaction with general practitioners' services. Br J Gen Pract. 1995;45(399):525-9.

14- Joolaee S, Hajibabaee F, Jafar Jalal E, Bahrani N. Assessment of Patient Satisfaction from Nursing Care in Hospitals of Iran University of Medical Sciences. Hayat. 2011;17(1):35-44. [Persian]

15- Khezri L, Ravaanipur M, Gahanpur F, Mirzai K. Assessment of patient satisfaction with nursing services provided in general wards of university hospitals in Bushehr 2012. Nurs J Vulnerable. 2015;2(3):36-46. [Persian]

16- Akhondzade R. Health system transformation project, an opportunity or a threat for doctors (Editorial). J Anaesthesiol Pain. 2014;5(1):1-2. [Persian]

17- Nazari R, Babaalipour Mouziraji F, Sharifnia H, Amini J, Akbari S. A comparative study on patient satisfaction with hospital services in Amol. J Urmia Nurs Midwifery Faculty. 2011;9(3):188-95. [Persian]

18- Hajinezhad MS, Rafii F, Jafarjalal E, Haghani H. Relationship between nurse caring behaviors from patients' perspectives \& their satisfaction. Iran J Nurs. 2007;20(49):73-83. [Persian]

19- Khamis K, Njau B. Patients' level of satisfaction on quality of health care at Mwananyamala hospital in Dar es Salaam, Tanzania. BMC Health Serv Res. 2014;14:400.

20- Gudarzian AH, Sharifnia H, Jafari H, Jamali S, Badiee $\mathrm{M}$, Sayemi $\mathrm{Z}$, et al. Inpatient satisfaction with health system transformation project in Mazandaran educational hospitals' Iran. J Mazandaran Univ Med Sci. 2016;26(136):190-5. [Persian]

21- Joolaee S, Jalili HR, Rafii F, Hajibabaee F, Haghani H. Relationship between moral distress and job satisfaction among nurses of Tehran University of Medical Sciences hospitals. Hayat. 2012;18(1):42-51. [Persian]

22- Jannati Y, Motlagh F, Kolbadinezhad N, Jafarnejad M. Patient satisfaction with nursing care in Behshahr public

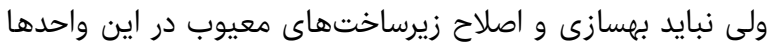

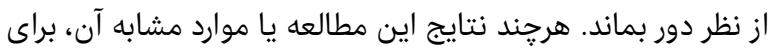

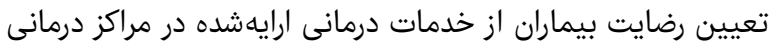

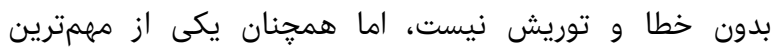

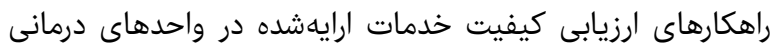

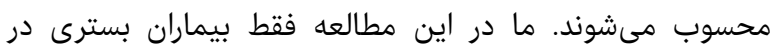

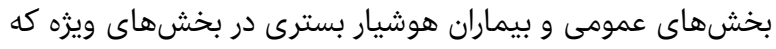

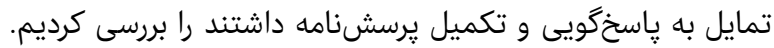

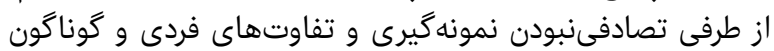

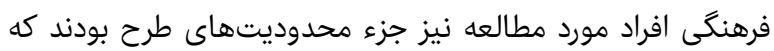

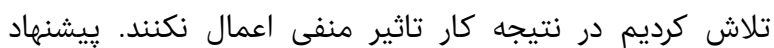

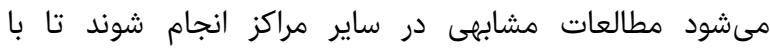

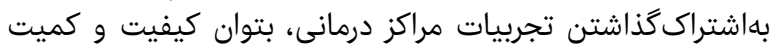
خدمات ارايهشده را ارتقا بخشيد لجربيات مرات<smiles>[As]=[AsH]</smiles>
وضعيت تاهل، ميزان تحصيلات و سن با ميزان رضايت بمندى

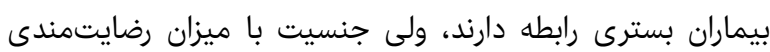
رابطه ندارد.

تشكر و قدردانى: در خاتمه بر خود فرض مىدانيم از كليه بيماران

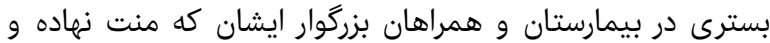

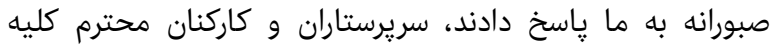

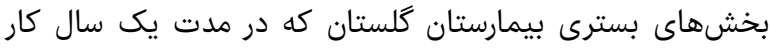

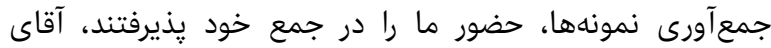

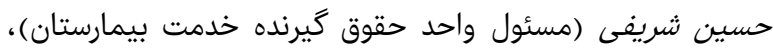

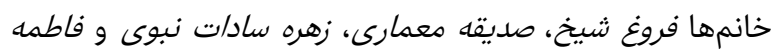

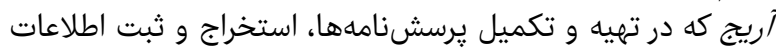

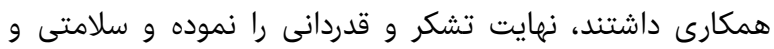

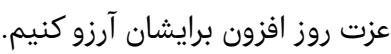

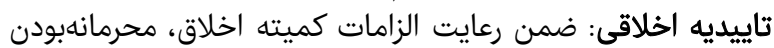

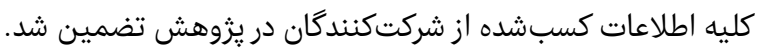

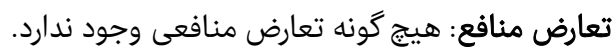

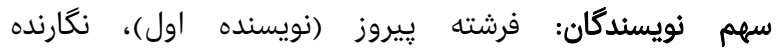

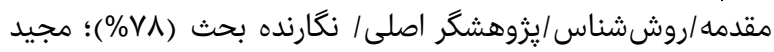

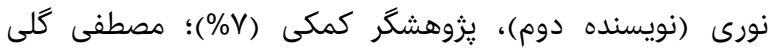

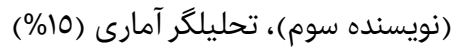

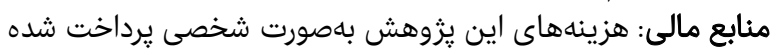
است.

\section{منابع}

1- Taylor C, Benger JR. Patient satisfaction in emergency medicine. Emerg Med J. 2004;21(5):528-32.

2- Smith MA, Schussler-Fiorenza C, Rockwood T. Satisfaction with care. In: Kane RL. Understanding health care outcomes research. 2nd Edition. Massachusetts: Jones \& Bartlett Learning; 2006.

3- Ebrahimnia M, Ameriun A, Azizabadi Farahani M, Khodami Vishte HR. Satisfaction rate of hospitalized

4- Farzadi F, Maftoun F. Quality management: Philosophy and trend. Payesh. 2002;1:57-59. [Persian]

5- Gunther M, Alligood MR. A discipline-specific determination of high quality nursing care. J Adv Nurs. 2002;38(4):353-9.

6- Azami A, Akbarzadeh K. Study of the satisfaction rate 
رضـ رضايتسنجى بيماران بسترى ازخدمات بهداشتى- درمانى ارايهده در بيمارستان كلستان تهران

Patients satisfaction of medical care delivered in

hospitals, Iran. J Mazandaran Univ Med Sci. outpatient clinics of endocrinology and metabolism in Tehran, Iran. Payesh. 2007;6(1):71-4. [Persian]

25- Sadjadian AS, Kaviani A, Younesian M, Fateh A.

Patients' Satisfaction with Services Provided at the Center for Breast Disease. Payesh. 2002;1(3):55-63. [Persian]

2016;26(143):252-6. [Persian]

23- Maftoon F, Farzadi F, Aeenparast A, Azin SA, Omidvari S, Jahangiri $\mathrm{K}$, et al. Who is responsible for health: Iranian Health Perception Survey (IHPS). Payesh. 2011;10(3):347-53. [Persian]

24- Khamseh ME, Aghili SR, Baradaran HR, Arabi A. 